\title{
The Flash-Lag, Fröhlich and Related Motion Illusions Are Natural Consequences of Discrete Sampling in the Visual System
}

\author{
Keith A. Schneider* \\ Department of Psychological and Brain Sciences, University of Delaware, Newark, DE, United States
}

The Fröhlich effect and flash-lag effect, in which moving objects appear advanced along their trajectories compared to their actual positions, have defied a simple and consistent explanation. Here, I show that these illusions can be understood as a natural consequence of temporal compression in the human visual system. Discrete sampling at some stage of sensory perception has long been considered, and if it were true, it would necessarily lead to these illusions of motion. I show that the discrete perception hypothesis, with a single free parameter, the perceptual moment or sampling rate, can quantitatively explain all of the scenarios of the Fröhlich and flash-lag effect. I interpret

OPEN ACCESS

Edited by:

Leila Reddy,

Université Toulouse III Paul Sabatier,

France

Reviewed by:

Niko Busch,

Universität Münster, Germany

Samson Chota,

Université Toulouse III Paul Sabatier,

France

*Correspondence:

Keith A. Schneider

keithas@udel.edu

Specialty section:

This article was submitted to

Perception Science,

a section of the journal

Frontiers in Psychology

Received: 14 February 2018

Accepted: 27 June 2018

Published: 31 July 2018

Citation:

Schneider KA (2018) The Flash-Lag, Fröhlich and Related Motion Illusions Are Natural Consequences of Discrete Sampling in the Visual System. Front. Psychol. 9:1227. doi: 10.3389/fpsyg.2018.01227 discrete perception as the implementation of data compression in the brain, and our conscious perception as the reconstruction of the compressed input.

Keywords: flash-lag effect, Fröhlich effect, motion illusions, discrete perception, temporal sampling

\section{INTRODUCTION}

The human visual system makes consistent errors localizing the positions of moving objects (Linares et al., 2009). In the Fröhlich effect (Fröhlich, 1923; Kerzel, 2010), the perceived initial positions of abruptly appearing moving objects are displaced along their trajectories from their actual onsets, as are, in the flash-lag effect (MacKay, 1958; Nijhawan, 1994), their positions compared to actually aligned static objects. Attribute changes occurring within moving objects are perceived as, similarly, displaced (Zeki and Moutoussis, 1997; Eagleman and Sejnowski, 2007). These motion illusions have defied coherent explanation, but I demonstrate that they can be simply explained as artifacts of discrete temporal subsampling in the visual system (Stroud, 1954). The illusions are a natural consequence of a discrete perception hypothesis wherein visual input is broken into discrete perceptual moments, with moving objects registered only in their final positions during each moment. The single parameter model, with perceptual moments lasting 100-150 ms, quantitatively accounts for the measured phenomenology of the illusions. The possibility of discrete perception has long been contemplated (James, 1890, 1909; Bergson, 1911; Stroud, 1954). Bergson (1911) likened our stream of consciousness to a motion picture and contended that perception was necessarily discrete, with our thoughts and memories operating upon static images. James (1890) initially rejected the notion of discrete perception, writing, "Consciousness... does not appear to itself chopped up in bits" (p. 239) and held that only our understanding was discrete: "... we take it in in discrete pulses of recognition" (p. 622). Later, however, James (1909), influenced by Bergson, changed positions, writing, "Time itself comes in drops" (p. 232). I show that the discrete perception hypothesis elegantly explains a large class of visual illusions. 


\section{METHODS AND RESULTS}

I hypothesize that visual input is sampled into discrete perceptual moments of duration $D$, and that any moving objects that might occupy a range of positions during the moment are registered at their final position occupied during each moment. The latter is a reasonable assumption, as motion deblurring techniques are thought to inhibit previous positions along a motion trajectory to prevent object persistence from confounding spatial extent (Burr, 1980), and the leading edge of a moving stimulus is most prominently evident in neural activity (Berry et al., 1999). We test this hypothesis by comparing its predictions to actual reports of visual perceptions of a variety of motion illusions.

In the Fröhlich effect (Figure 1), when the moving object appears, its first location is not registered until the end of the current perceptual moment. Because the perceptual moments are randomly positioned in time relative to stimuli, during a moment with duration $D$, the last position of the moving object will be displaced on average by a time $D / 2$ along its trajectory. The first observed position is therefore displaced from its actual position by the distance traveled by the object during this time, and the remaining trajectory of the object is reconstructed from this starting point. Typical estimates of $D / 2$ for the Fröhlich effect are about $50 \mathrm{~ms}$ (Kerzel, 2010), yielding $D \approx 100 \mathrm{~ms}$.

For the flash-lag illusion (Figure 2), the size of the effect observed on each trial of an experiment again is determined by the random temporal phase of the perceptual moment in relation to the stimuli. The size of the flash-lag should be uniformly distributed on the interval $[0, D]$. Both the flash and moving object are sampled in the same manner - the flash too is perceived at its final position within a moment, but its position is static, and its onset time and duration within the moment do not affect its perception. If the flash occurs at the last instant of the perceptual moment, and thus, the final position of the moving object within the moment is actually aligned with the flash onset, the flash-lag would be 0 . If the flash onset occurs at the very beginning of a moment, the position of the moving object would be perceived at a time $D$ later. The predicted mean magnitude of the flashlag effect is $D / 2$, which has been measured as $60.1-77.5 \mathrm{~ms}$ (Murakami, 2001). The discrete perception hypothesis makes consistent predictions for both the continual motion version of the flash-lag effect, above, for which the moving object continues to move along its trajectory both before and after the flash, as well as the flash initiated condition (Khurana and Nijhawan, 1995; Eagleman and Sejnowski, 2000b), in which the flashed object and the moving object both onset simultaneously. Note that the timing of the onsets of the flash and moving object is the same (Aschersleben and Müsseler, 1999; Eagleman and Sejnowski, 2000a; Arnold et al., 2009), as they appear within the same moment-only the position of the moving object is recorded at the end of the moment and is thus perceived as advanced from its initial position along its trajectory. For the flash terminated condition, in which the moving object halts at the same instant the flash appears, no misalignment between the flash and moving object is predicted, or observed experimentally (Eagleman and Sejnowski, 2000b; Rizk et al., 2009), since the last position of the moving object in any moment necessarily
A

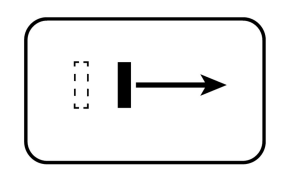

B Actual stimulus

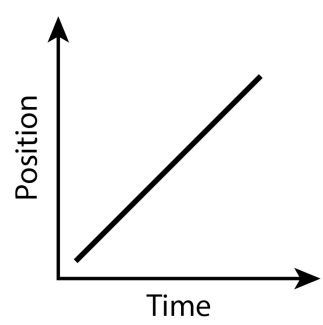

C Perceived stimulus

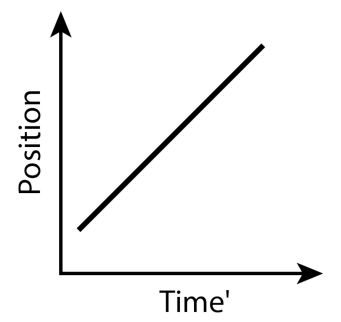

E Reconstructed stimulus

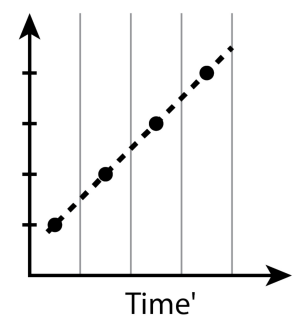

FIGURE 1 | The Fröhlich effect. (A) A moving bar appears with an initial position indicated by the dashed outline, but the perceived initial position is displaced along the trajectory. Space-time diagrams (B-E). (B) An object (black line) abruptly appears and begins moving. (C) The perception of this situation is that the object first appears in a position displaced along the trajectory of motion from the actual initial position. Note that the perceived 'Time' axis is delayed by some amount relative to the actual Time in (B). (D) The stimulus is sampled into discrete time windows, bounded by the vertical gray lines. The position of the moving object within each window is registered as the final position of the object in the window, indicated by the black dots. (E) The recorded positions of the moving object in time, indicated by the black dots, represent the position of the object at each time window. The smooth conscious perception of the moving object is reconstructed or interpolated between the discrete registered positions, as indicated by the dashed line.

is aligned with the flash (because there is no motion after the flash).

An interesting case for the flash-lag effect occurs when the moving object abruptly reverses direction (Whitney and Murakami, 1998). Until time $D$ before the reversal, there is no effect of the reversal on the perceived position or the moving object, which appears to lead the flash by time $D / 2$ as usually. However, by time $D / 2$ before the reversal, the average position of the moving object should be perceived as aligned with the flash, and by the time of the reversal, the average perceived position at the end of the moment would be $D / 2$ back in the reversed direction. The average position should smoothly transition among these points, which is indeed observed experimentally (Whitney and Murakami, 1998; Brenner and Smeets, 2000; Whitney et al., 2000; Eagleman and Sejnowski, 2000b).

A variant of the flash-lag effect has been termed the flash-jump effect (Eagleman and Sejnowski, 2007), which is also similar to the 
A

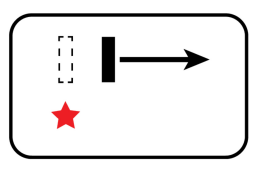

B Actual stimulus

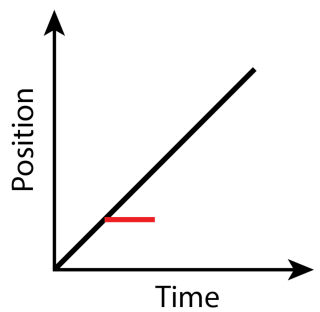

C Perceived stimulus

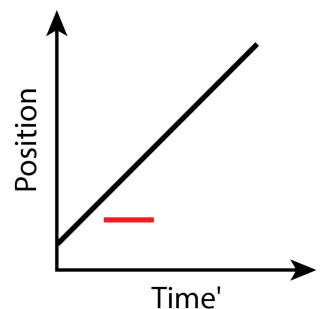

Time'
D Sampled stimulus

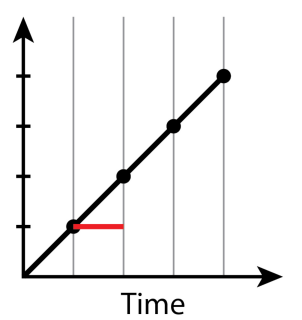

E Reconstructed stimulus

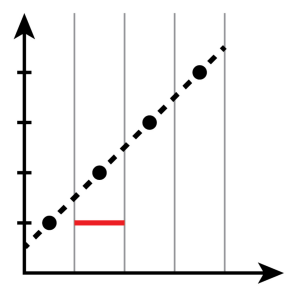

Time'

FIGURE 2 | The flash-lag effect. (A) A flash (red star) occurs aligned with a moving bar (dashed outline), but the bar at the time of the flash is perceived as advanced along its trajectory. Space-time diagrams (B-E). (B) An object (black line) is continuously moving through the visual field. At some time, a new static object (red line) flashes in alignment with the moving object. Note that the onset and duration of the flash within the moment do not affect its perception; the flash is thus depicted as filling the moment. (C) The perceived location of the flash appears to lag in behind the moving object. (D,E) as in Figure 1 .

color-motion asynchrony illusion (Zeki and Moutoussis, 1997). Here, the moving object abruptly changes one of its attributes, e.g., its color, during its motion trajectory, and the change is perceived, not in its veridical position, but rather displaced along the trajectory of motion. This is a natural consequence of the perceptual moments. The attribute change occurs during one perceptual moment, and the position of the moving object is registered in that moment as its last position within the moment. Therefore, the attribute change should be observed on average at a time $D / 2$ past its actual time of occurrence.

Perhaps the most comprehensive flash-lag data set was collected by Ikuya Murakami using random motion (Murakami, 2001), which probes the flash-lag effect on a fine time scale and intrinsically includes all of the motion reversal scenarios. Any model of the flash-lag effect needs to be able to explain the time course of these data, rather than just the mean magnitude of the effect that is typically reported in other studies. In this experiment, bars of fixed durations $(127.5,167$, or $255 \mathrm{~ms}$ in separate experiments) were presented in random positions. Periodically, flashes $(8.5 \mathrm{~ms}$ duration) were presented, and subjects had to indicate whether they perceived the flash to the left or right of each bar. Under the discrete perception hypothesis,

the perceived position of the bar during each perceptual moment is its position at the end of the moment. Therefore, if the location of the bar when the flash occurs has not changed by the end of the moment, the subject will report the "correct" relative position. However, if the bar has moved to a different random position by the end of the moment, the subject's judgment will be at chance. I sought to quantitatively fit the discrete perception model to this rich data set, which I obtained directly from Ikuya Murakami (Figures 3-5 for the three separate subjects). To fit the data, we must determine the probability that the bar position at any instant in time is the same as the bar position at the end of the moment in which the flash occurred. Let $\Delta F$ be the onset time of the bar subtracted from the onset time of the flash (the $x$-axis in Figures 3-5), $t_{\mathrm{F}}$ the time of the flash, $t_{\mathrm{F}}-\Delta F$ the onset time of the bar, $t_{\mathrm{F}}-\Delta F-B$ the bar offset time, where $B$ is the duration of the bar. Since the perceptual moment is randomly aligned in time relative to the flash, the end of the moment occurs at $t_{\mathrm{F}}+D \varphi$, where $D$ is the duration of the moment and $\varphi \in[0,1]$ is the random phase. For a given $\Delta F$, we need to determine the probability that the bar onset time occurs before the end of the moment, i.e., $-\Delta F<D \varphi$, and that the bar offset occurs at or after the end of the moment, i.e., $B-\Delta F \geq D \varphi$. $D$ can be determined for each subject by sampling or integrating over $\varphi$ and minimizing the error between the model and data. In principle, $D$ could be drawn from any distribution, but the results show that this model, with a single uniform parameter $D$, linked among all of the three bar durations, adequately accounts for the data, yielding $D=152.5,200.3$, and $150.0 \mathrm{~ms}$ for the three subjects, respectively (the second subject with the largest $D$, had quite noisy data compared to the other two). For comparison, I also fit a two-parameter model, with $D$ drawn from a normal distribution. Figures 3-5 show the data for the three subjects, for the three separate bar durations. The red line is the fit for a uniform moment duration, and the green line is the best fit for $D$ with a normally distributed duration (see insets). The discrete perception model accounts for the data simply and elegantly, whereas other models such as motion extrapolation and differential latency fail to fit the data or require modification (e.g., multiple differential latencies) (Murakami, 2001). Any model that involves a single timing or position differential cannot account for the ramps evident in the data and fits in Figures 3-5, which are fit by the sliding time window in the discrete perception model. This model needs to be tested on additional time-resolved data sets, in future experiments.

\section{DISCUSSION}

The leading explanations of these illusions are the differential latency (Whitney and Murakami, 1998) and postdiction (Eagleman and Sejnowski, 2000b) hypotheses. Both are approximations of the discrete perception hypothesis but do not completely explain all of the phenomenology. The differential latency hypothesis suggests that moving stimuli have a temporal processing advantage over unexpected or flashed stimuli and thus appear to lead them in time. The hypothesis is unable to 


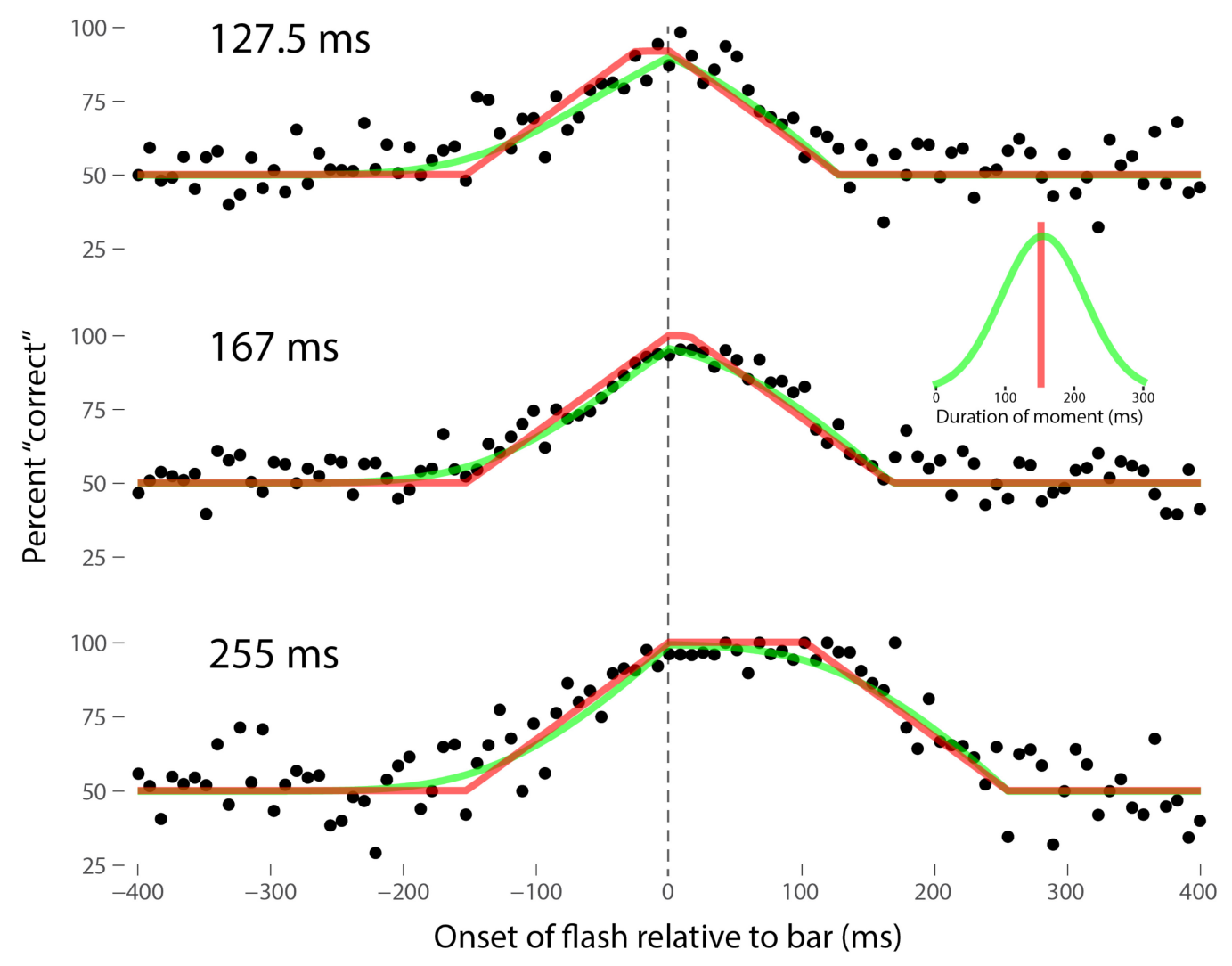

FIGURE 3 | A fit of the discrete perception model to the random flash-lag data (Murakami, 2001). In this study, a bar was randomly displaced $\pm 3^{\circ}$ horizontally about fixation every 127.5 (top), 167 (middle), or 255 ms (bottom). An independent brief (8.5 ms) flash occurred every $3 \pm 1 \mathrm{~s}$, within \pm 50 ' of fixation. The observers' task was to report whether the flash occurred to the left or right of fixation. The data above were registered to the onset of the bar. The observers' responses were scored as "correct" relative to this bar, as opposed to a subsequent or previous bar. The black points are averages from multiple trials for one subject (IM). The red lines are the fit of the single-parameter discrete perception model, linked across the three different bar durations, with $D=152.5$ ms for this subject. The green lines indicated the best fit for $D$ with a variable duration, distributed normally, as shown in the inset ( $\mu=154 \mathrm{~ms}, \sigma=61 \mathrm{~ms}$ ). The model shows the probability that this bar will be the one present at the end of the perceptual moment, averaged over all of the randomly placed possible phases of the moment.

explain the flash-initiated condition of the flash-lag effect, in which both the flash and moving object appear simultaneously, or the Fröhlich and the flash-jump effects, which have only one object and thus no possible latency differential. Moreover, temporal order judgments do not reveal temporal advantages for moving objects (Aschersleben and Müsseler, 1999; Eagleman and Sejnowski, 2000a; Arnold et al., 2009), nor is there any physiological evidence for such an advantage (Arnold et al., 2009).

The postdiction hypothesis (Eagleman and Sejnowski, 2000b) suggests that the position of a moving object is integrated for approximately $80 \mathrm{~ms}$ after a flashed object, to compare their positions. Similar to the discrete perception hypothesis, the postdiction hypothesis identifies the origins of motion illusions as spatial and not temporal, i.e., the position of the moving object is misconstrued at the time of the flash. It can explain the many variations of the flash-lag effect but has difficulty explaining the flash-jump and color-motion asynchrony illusions, because the flash itself is seen at a different location, and cannot account for the variability of the effects (Linares et al., 2009), which is naturally explained by the discrete perception hypothesis as the random phase alignment of the perceptual moments with the visual stimuli. It also seems unlikely that a flash would reset the integration period (Krekelberg and Lappe, 2000): triggering could work for isolated events, but it does not seem plausible for a continuous visual scene, although eye movements could be responsible for triggering (Meister, 1951). Triggered vs. independent models have been tested for temporal order judgments. Sternberg and Knoll (1973) proposed a triggered-moment model, in which the perceptual moment was triggered by a stimulus, and a perceptual-moment model, in which the timing of perceptual moments is independent of stimuli. Although, the predictions of the two models are similar, Schneider and Bavelier (2003) found that the perceptual-moment better explained data from temporal order and simultaneity judgments.

A revision of the postdiction theory (Eagleman and Sejnowski, 2007) suggests that moving objects are perceived to be biased in advanced positions along their trajectoriesthis is an accurate summary of the phenomenology, and the discrete perception hypothesis explains the underlying mechanism. Other studies have suggested temporal sampling 
Schneider

Discrete Perception Generates Motion Illusions

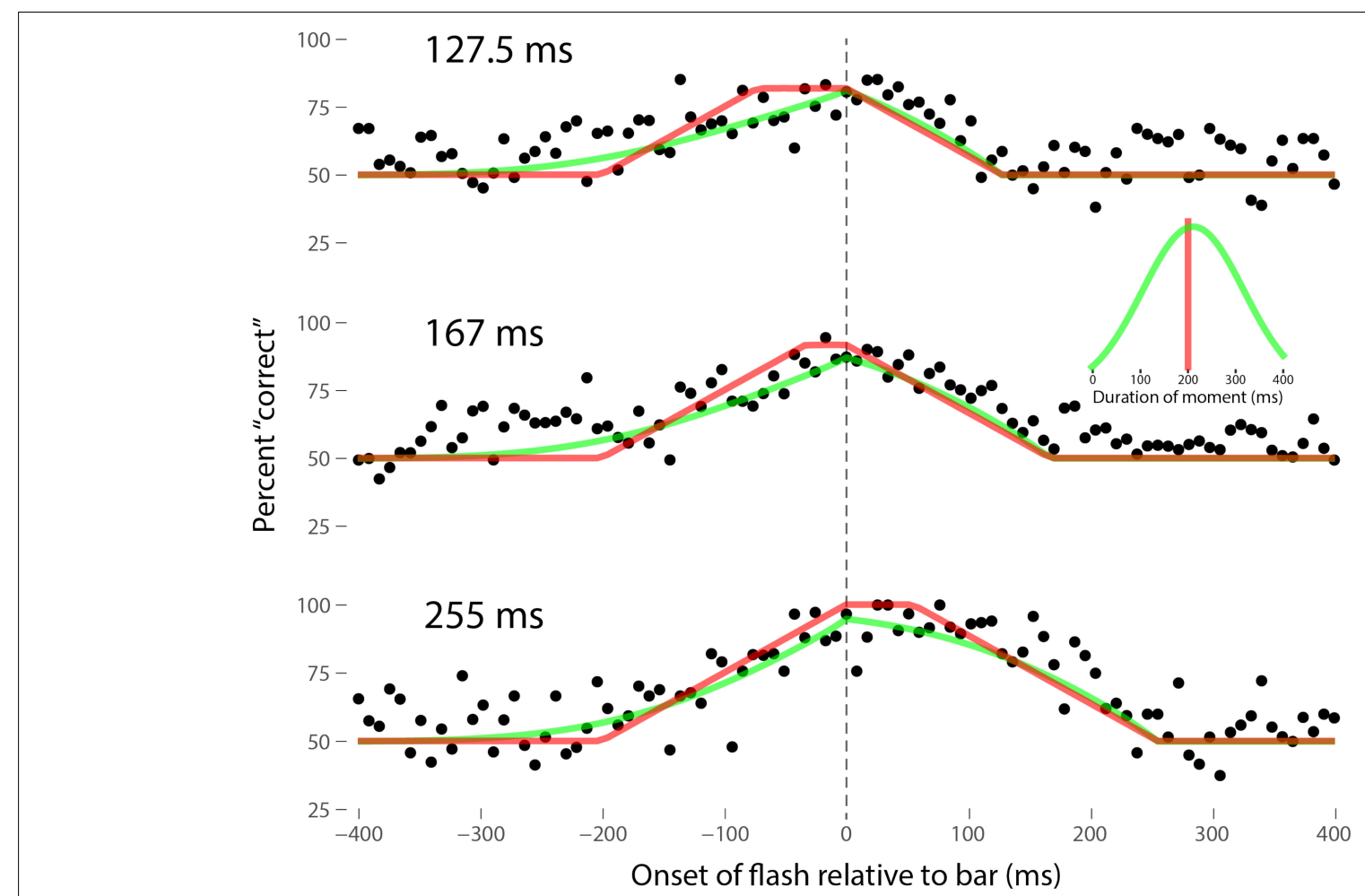

FIGURE 4 | Data for the second subject, MM, as per Figure 3. Here $D=200.3 \mathrm{~ms}$ for the single-parameter model. For the two-parameter model with $D$ normally distributed, $\mu=212 \mathrm{~ms}$ and $\sigma=106 \mathrm{~ms}$, as shown in the inset.

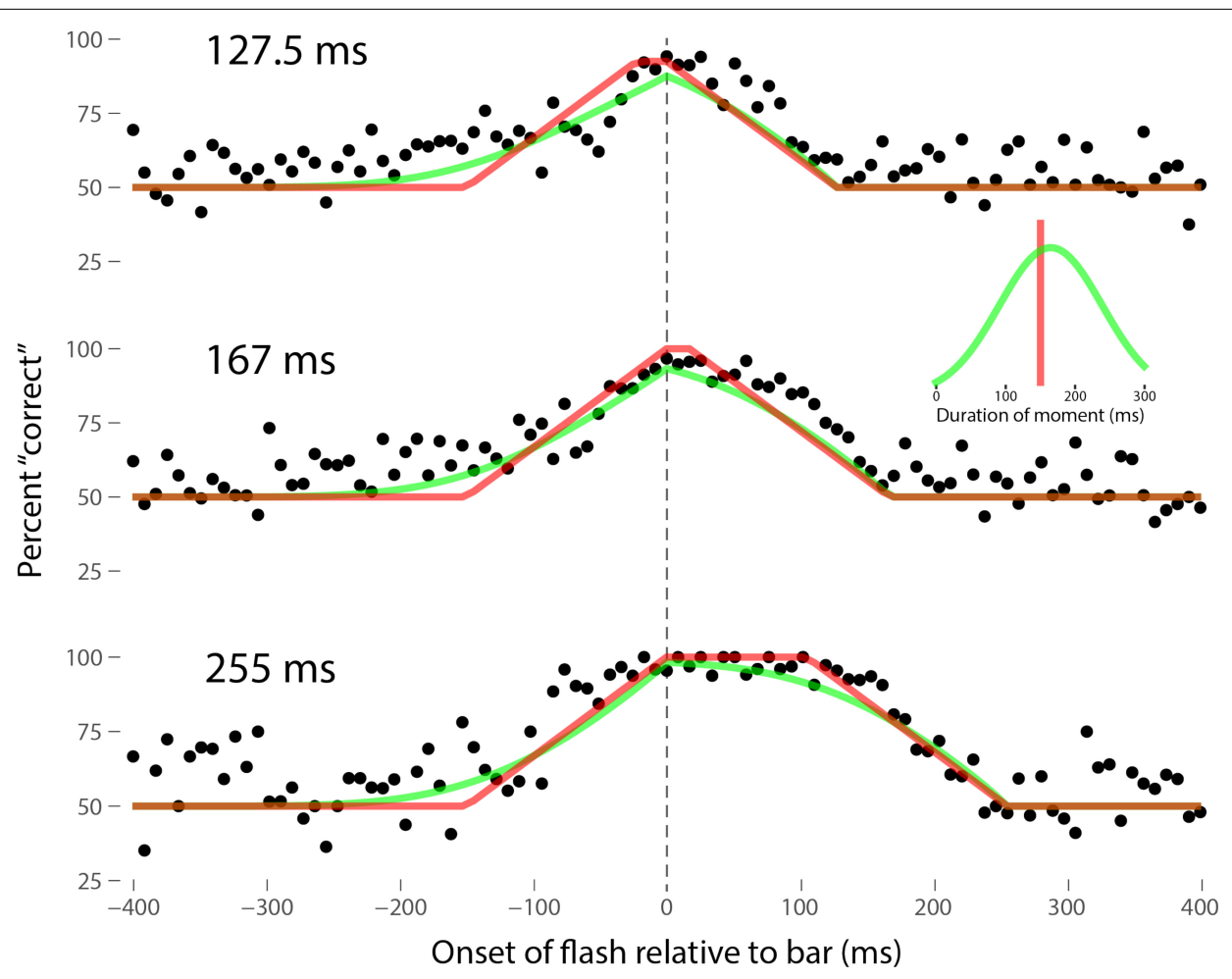

FIGURE $\mathbf{5}$ | Data for the third subject, SN, as per Figure 3. Here $D=150.0 \mathrm{~ms}$ for the single-parameter model. For the two-parameter model with $D$ normally distributed, $\mu=165 \mathrm{~ms}$ and $\sigma=74 \mathrm{~ms}$, as shown in the inset.

Frontiers in Psychology | www.frontiersin.org

5

July 2018 | Volume 9 | Article 1227 
(Brenner and Smeets, 2000) or positional averaging that is weighted toward the most recently sampled position (Roulston et al., 2006), explanations that are compatible with the discrete perception hypothesis. Another study claimed to be able to explain the flash-lag effect in terms of a bias to perceive certain velocities (Wojtach et al., 2008). They showed that the flash-lag effect was not linear with velocity, as other studies had showed, but rather was a logarithmic function, and they showed that this same function would result from the distribution of velocities that would be projected upon the retina due to the distribution of three dimensional motions in the natural world. However, this study does not provide an explanation for the flash-lag effect, but rather an explanation for biases in motion velocity; since the flash-lag effect is velocity dependent, it will reflect any biases in velocity perception.

One common observation, for both the Fröhlich effect and the variations of the flash-lag effect, is that attention can reduce the size of the effects (Müsseler and Aschersleben, 1998; Brenner and Smeets, 2000; Baldo and Namba, 2002; Namba and Baldo, 2004; López-Moliner and Linares, 2006; Sarich et al., 2007; Shioiri et al., 2010), though exceptions have been reported (Khurana et al., 2000). Since this occurs for suprathreshold stimuli, one should avoid explanations that involve changes in perceptual thresholds. Rather, I hypothesize that attention operates to enhance the available information by increasing the sampling rate (Sarich et al., 2007; Samaha et al., 2015; Samaha and Postle, 2015; Wutz et al., 2018), which need not occur uniformly throughout the visual field, thereby shortening the perceptual moment and diminishing the corresponding effect magnitudes. A similar variation in the duration of the perceptual moment was proposed for stimulus intensity (Shallice, 1964). The measured size of the perceptual moment does vary considerably among individuals and among different tasks.

Much of the spatiotemporal input to the visual system is redundant. Retinal circuits remove spatial redundancy, and this process continues in the cortex (Olshausen and Field, 1996), akin to compression techniques used to reduce the file size of digital images. The temporal mechanisms used by the brain are less clear (Nortmann et al., 2015), but temporal compression necessarily involves temporal subsampling. Note that low-pass filtering is theoretically equivalent to subsampling-a cubic spline for example can be exactly described by three discrete samples. Such discrete perception has long been debated (James, 1890, 1909; Bergson, 1911; Stroud, 1954), suggesting that the brain processes snapshots of the visual world similar to the frames of a motion picture, but has never been definitively proven. The hypothesis of discrete perception was perhaps most clearly articulated by Stroud (1954), and recently has been championed by VanRullen et al. (2010, 2014) and VanRullen and Koch (2003) to explain the continuous wagon wheel illusion (Purves et al., 1996). Stroud described "moments" of perception with a duration of approximately $100 \mathrm{~ms}$, during which any events recorded by the visual system could not be temporally differentiated. However, his description did not precisely specify how events occurring during each moment were registered, making predictions from his theory difficult.
The discrete perception hypothesis described herein is agnostic as to the distribution of the frame rates-this is an empirical question. An examination in the distribution of subject responses could, for example, rule out a constant frame rate, but it would be more difficult to distinguish variable frame rate models from other perceptual hypotheses.

Our conscious perceptions of the world are fluid, not discrete; if we sample the world discretely, our continuous subjective perceptions must then be reconstructed from these samples. I propose that sampling/compression occurs during a feedforward processing cycle, terminating in object recognition, and that our conscious perceptions result from reconstruction/decompression during a feedback cycle. Since the reconstruction is based on sampled input, any temporal artifacts arising from the sampling process will be carried through to perception. Smoothing or interpolation between discrete frames would explain apparent motion as well as the phi phenomenon in which color changes of a moving object are perceived before they actually occur (Kolers and von Grünau, 1976). Interpolation could, similarly, explain the motion vectoring effects reported by Eagleman and Sejnowski (Eagleman and Sejnowski, 2007), in which moving objects or colors were observed in locations where they were not ever present. Perhaps the electrical alpha rhythms in the brain reflect the compression/decompression process, though their phase has at most small perceptual consequences (VanRullen et al., 2014), likely because the discrete perceptual moments completely tile the input space with no gaps among them. Previously, the alpha rhythms have been correlated with the magnitude of the flashlag effect on each trial (Chakravarthi and Vanrullen, 2012), which is parsimonious with the present theory, and perhaps may provide a neural substrate for discrete sampling. Area MT is a likely candidate to perform a motion decompression function, as conscious perception is abolished if the feedback link from MT to V1 is disrupted (Pascual-Leone and Walsh, 2001), and if MT is damaged bilaterally, stroboscopic perception can result (Zihl et al., 1983), as if only the key frames in a compressed video were perceived.

\section{CONCLUSION}

\section{In 1869, Tolstoy (1869/2005):}

For the human mind absolute continuity of motion is inconceivable. The laws behind any motion become comprehensible to man only when he breaks that motion down into arbitrary selected units and subjects these to examination. But at the same time this arbitrary sub-division of continuous motion into discontinuous units is the cause of much human error. (Vol. 3, Part 3, Ch. 1).

Such is the case in perception, where a discrete analysis of the continuous visual stream causes spatiotemporal misrepresentations that manifest as illusions of motion.

\section{AUTHOR CONTRIBUTIONS}

The author confirms being the sole contributor of this work. 


\section{REFERENCES}

Arnold, D. H., Ong, Y., and Roseboom, W. (2009). Simple differential latencies modulate, but do not cause the flash-lag effect. J. Vis. 9, 1-8. doi: 10.1167/ 9.5.4

Aschersleben, G., and Müsseler, J. (1999). Dissociations in the timing of stationary and moving stimuli. J. Exp. Psychol. Hum. Percept. Perform. 25, 1709-1720. doi: 10.1037/0096-1523.25.6.1709

Baldo, M. V. C., and Namba, J. (2002). The attentional modulation of the flash-lag effect. Braz. J. Med. Biol. Res. 35, 969-972. doi: 10.1590/S0100879X2002000800014

Bergson, H. (1911). Creative Evolution. New York, NY: Henry Holt and Company.

Berry, M. J., Brivanlou, I. H., Jordan, T. A., and Meister, M. (1999). Anticipation of moving stimuli by the retina. Nature 398, 334-338. doi: 10.1038/ 18678

Brenner, E., and Smeets, J. B. J. (2000). Motion extrapolation is not responsible for the flash-lag effect. Vision Res. 40, 1645-1648. doi: 10.1016/S0042-6989(00) 00067-5

Burr, D. (1980). Motion smear. Nature 284, 164-165. doi: 10.1038/28 $4164 \mathrm{a} 0$

Chakravarthi, R., and Vanrullen, R. (2012). Conscious updating is a rhythmic process. Proc. Natl. Acad. Sci. U.S.A. 109, 10599-10604. doi: 10.1073/pnas. 1121622109

Eagleman, D. M., and Sejnowski, T. J. (2000a). Flash-lag effect: differential latency, not postdiction. Science 290:1051. doi: 10.1126/science.290.5494. $1051 \mathrm{a}$

Eagleman, D. M., and Sejnowski, T. J. (2000b). Motion integration and postdiction in visual awareness. Science 287, 2036-2038.

Eagleman, D. M., and Sejnowski, T. J. (2007). Motion signals bias localization judgments: a unified explanation for the flash-lag, flashdrag, flash-jump, and Fröhlich illusions. J. Vis. 7, 1-12. doi: 10.1167/ 7.4 .3

Fröhlich, F. W. (1923). Über die messung der empfindungszeit. Z. Sinnesphysiol. $54,58-78$.

James, W. (1890). The Principles of Psychology. New York, NY: Henry Holt.

James, W. (1909). A Pluralistic Universe. New York, NY: Longmans, Green, and Co.

Kerzel, D. (2010). “The Fröhlich effect: past and present," in Space and Time in Perception and Action, eds R. Nijhawan and B. Khurana (Cambridge: Cambridge University Press), 321-337. doi: 10.1017/CBO97805117505 40.019

Khurana, B., and Nijhawan, R. (1995). Extrapolation or attention shift? Nature 378:566. doi: 10.1038/378566a0

Khurana, B., Watanabe, K., and Nijhawan, R. (2000). The role of attention in motion extrapolation: are moving objects "corrected" or flashed objects attentionally delayed? Perception 29, 675-692. doi: 10.1068/ p3066

Kolers, P. A., and von Grünau, M. (1976). Shape and color in apparent motion. Vision Res. 16, 329-335. doi: 10.1016/0042-6989(76) 90192-9

Krekelberg, B., and Lappe, M. (2000). The position of moving objects. Science 289:1107. doi: 10.1126/science.289.5482.1107a

Linares, D., Holcombe, A. O., and White, A. L. (2009). Where is the moving object now? Judgments of instantaneous position show poor temporal precision (SD $=70 \mathrm{~ms}$ ). J. Vis. 9, 1-14. doi: 10.1167/9.13.9

López-Moliner, J., and Linares, D. (2006). The flash-lag effect is reduced when the flash is perceived as a sensory consequence of our action. Vision Res. 46, 2122-2129. doi: 10.1016/j.visres.2005.11.016

MacKay, D. M. (1958). Perceptual stability of a stroboscopically lit visual field containing self-luminous objects. Nature 181, 507-508. doi: 10.1038/181 $507 \mathrm{a} 0$

Meister, R. K. (1951). A Hypothesis Concerning the Function of the Occipital Alpha Rhythm in Vision. Department of Psychology. Ph.D. thesis, University of Chicago, Chicago, IL.

Murakami, I. (2001). A flash-lag effect in random motion. Vision Res. 41, 3101-3119. doi: 10.1016/S0042-6989(01)00193-6

Müsseler, J., and Aschersleben, G. (1998). Localizing the first position of a moving stimulus: the Fröhlich effect and an attention-shifting explanation. Percept. Psychophys. 60, 683-695. doi: 10.3758/BF0320 6055

Namba, J., and Baldo, M. V. C. (2004). The modulation of the flashlag effect by voluntary attention. Perception 33, 621-631. doi: 10.1068/ p5212

Nijhawan, R. (1994). Motion extrapolation in catching. Nature 370, 256-257. doi: 10.1038/370256b0

Nortmann, N., Rekauzke, S., Onat, S., König, P., and Jancke, D. (2015). Primary visual cortex represents the difference between past and present. Cereb. Cortex 25, 1427-1440. doi: 10.1093/cercor/bht318

Olshausen, B. A., and Field, D. J. (1996). Emergence of simple-cell receptive field properties by learning a sparse code for natural images. Nature 381, 607-609. doi: $10.1038 / 381607 \mathrm{a} 0$

Pascual-Leone, A., and Walsh, V. (2001). Fast backprojections from the motion to the primary visual area necessary for visual awareness. Science 292, 510-512. doi: 10.1126/science.1057099

Purves, D., Paydarfar, J. A., and Andrews, T. J. (1996). The wagon wheel illusion in movies and reality. Proc. Natl. Acad. Sci. U.S.A. 93, 3693-3697. doi: 10.1073/ pnas.93.8.3693

Rizk, J. K., Chappell, M., and Hine, T. J. (2009). Effect of motion smoothness on the flash-lag illusion. Vision Res. 49, 2201-2208. doi: 10.1016/j.visres.2009. 06.010

Roulston, B. W., Self, M. W., and Zeki, S. (2006). Perceptual compression of space through position integration. Proc. Biol. Sci. 273, 2507-2512. doi: 10.1098/rspb. 2006.3616

Samaha, J., Bauer, P., Cimaroli, S., and Postle, B. R. (2015). Top-down control of the phase of alpha-band oscillations as a mechanism for temporal prediction. Proc. Natl. Acad. Sci. U.S.A. 112, 8439-8444. doi: 10.1073/pnas.150368 6112

Samaha, J., and Postle, B. R. (2015). The speed of alpha-band oscillations predicts the temporal resolution of visual perception. Curr. Biol. 25, 2985-2990. doi: 10.1016/j.cub.2015.10.007

Sarich, D., Chappell, M., and Burgess, C. (2007). Dividing attention in the flash-lag illusion. Vision Res. 47, 544-547. doi: 10.1016/j.visres.2006. 09.029

Schneider, K. A., and Bavelier, D. (2003). Components of visual prior entry. Cogn. Psychol. 47, 333-366. doi: 10.1016/S0010-0285(03) 00035-5

Shallice, T. (1964). The detection of change and the perceptual moment hypothesis. Br. J. Stat. Psychol. 17, 113-135. doi: 10.3758/s13414-0150963-9

Shioiri, S., Yamamoto, K., Oshida, H., Matsubara, K., and Yaguchi, H. (2010). Measuring attention using flash-lag effect. J. Vis. 10, 1-13. doi: 10.1167/ 10.10.10

Sternberg, S., and Knoll, R. L. (1973). "The perception of temporal order: fundamental issues and a general model," in Attention and Performance IV, ed. S. Kornblum (New York, NY: Academic Press), 629-685.

Stroud, J. M. (1954). "The fine structure of psychological time," in Information Theory in Psychology, ed. H. Quastler (Glencoe, IL: Free Press), 174-205.

Tolstoy, L. (1869/2005). War and Peace, A. Briggs, trans. New York, NY: Penguin Group.

VanRullen, R., and Koch, C. (2003). Is perception discrete or continuous? Trends Cogn. Sci. 7, 207-213.

VanRullen, R., Reddy, L., and Koch, C. (2010). "A motion illusion reveals the temporally discrete nature of visual awareness," in Space and Time in Perception and Action, eds R. Nijhawan and B. Khurana (Cambridge: Cambridge University Press), 521-535.

VanRullen, R., Zoefel, B., and Ilhan, B. (2014). On the cyclic nature of perception in vision versus audition. Philos. Trans. R. Soc. Lond. B Biol. Sci. 369:20130214. doi: 10.1098/rstb.2013.0214

Whitney, D., and Murakami, I. (1998). Latency difference, not spatial extrapolation. Nat. Neurosci. 1, 656-657. doi: 10.1038/ 3659

Whitney, D., Murakami, I., and Cavanagh, P. (2000). Illusory spatial offset of a flash relative to a moving stimulus is caused by differential latencies for moving and flashed stimuli. Vision Res. 40, 137-149. doi: 10.1016/S0042-6989(99) 00166-2 
Wojtach, W. T., Sung, K., Truong, S., and Purves, D. (2008). An empirical explanation of the flash-lag effect. Proc. Natl. Acad. Sci. U.S.A. 105, 16338-16343. doi: 10.1073/pnas.0808916105

Wutz, A., Melcher, D., and Samaha, J. (2018). Frequency modulation of neural oscillations according to visual task demands. Proc. Natl. Acad. Sci. U.S.A. 115, 1346-1351. doi: 10.1073/pnas.1713318115

Zeki, S., and Moutoussis, K. (1997). Temporal hierarchy of the visual perceptive systems in the Mondrian world. ProcBiol. Sci. 264, 1415-1419. doi: 10.1098/ rspb.1997.0197

Zihl, D., von Cramon, D., and Mai, N. (1983). Selective disturbance of movement vision after bilateral brain damage. Brain 106, 313-340. doi: 10.1093/brain/106. 2.313
Conflict of Interest Statement: The author declares that the research was conducted in the absence of any commercial or financial relationships that could be construed as a potential conflict of interest.

The reviewer SC and the handling editor declared their shared affiliation.

Copyright (c) 2018 Schneider. This is an open-access article distributed under the terms of the Creative Commons Attribution License (CC BY). The use, distribution or reproduction in other forums is permitted, provided the original author(s) and the copyright owner(s) are credited and that the original publication in this journal is cited, in accordance with accepted academic practice. No use, distribution or reproduction is permitted which does not comply with these terms. 